H. T., J. Endocrin. 22, 195 (1961). - 29. Makesh, V. B., R. B. Greenblatt, C. K. Aydar, S. Roy, R. A. Puebla and J. O. Ellegood, J. Clin. Endocrin. 24, 1283 (1964). - 30. Haiman, B. L. and M. M. Martin, J. Clin. Endocrin. 24, 1195 (1964). 31. Kappas, A. and T. F. Gallagher, J. Clin. Invest. 34, 1566
(1955). - 32. Beas, F., R. P. Zurbrugg, J. Cara and I. I. Gardner, J. Clin. Endocrin. 22, 1090 (1962). - 33. Starnes, W. R., T. F. PartLow, M. C. Grammer, L. Kornel and S. R. Hrll, Analy. Biochem. 6, 82 (1963). - 34. Brooksbank, B. W. L. and A. Salokangas, Acta endrocr., K'hvn 30, 231 (1959).

\author{
Doz. Dr. L. E. Böttiger \\ King Gustaf Vth Research Institute \\ Stockholm, Schweden
}

Dr. B. T. Lisboa

Karolinska Sjukhuset

Stockholm 60/Schweden

\title{
Die Problematik der GoRdonschen Testsubstanz
}

\author{
Von H.-J. Lieschke, H. Hartmann, J. Löbe, H. Кoch und K. Seige
}

Auls der Medizinischen Universitätsklinik Leipzig (Direktor:Prof. Dr. R. Em mirich), der Radiologischen Universitätsklinik Leipzig (Direktor: Prof. Dr. W. Oelßner) und dem Institut für angewandte Radioaktivität (elem. Direktor: Prof. Dr. C. F. Weiss) der Deutschen Akademie der Wissenscbaften zu Berlin

(Eingegangen am 26. September 1966)

Es wird auf die Instabilität der GoRDon'schen Testverbindung (,PVP-T_131 J“) zum Nachweis der Exsudativen Enteropathie hingewiesen und über die Ergebnisse von Stuhl- und Urindialysen berichtet.

Danach besteht die Aktivität des Urins zu 90\% aus abgespaltenem ionogenen ${ }^{131}$ Jod, die fäkkale Aktivität zu mindestens 30-50\% aus freiem ionogenen ${ }^{131} \mathrm{Jod}$. Als Modellsubstanz für den enteralen Eiweißverlust ist die Substanz daher nicht geeignet. - Die Ursache der erhöhten Abspalterate in vivo sowie der Alterung des Präparates in vitro dürfte in der einfachen Anlagerung des ${ }^{131}$ Jods an PVP-Doppelbindungen zu suchen sein. Die von Gordon angenommene aromatische Bindung des ${ }^{131}$ Jod am PVP-T wird abgelehnt.

The instability of Gordon's test compound, "PVP-T_131I", for the diagnosis of exudative enteropathy was studied, and results from the dialysis of faeces and urine are reported.

Free, ionisable ${ }^{131}$ i is responsible for up to $90 \%$ of the activity of the urine and at least $30-50 \%$ of the faeces. Thus the compound cannot be used for the measurement of the enteric loss of protein. - The increased rate of cleavage in vivo and the ageing of the preparation in vitro is caused by the simple transfer of ${ }^{131} \mathrm{I}$ to the unsaturated bonds of PVP. Gordon was therefore wrong in assuming that ${ }^{131} \mathrm{I}$ is bound by an aromatic linkage in PVP-T.

Die Verwendung von jodmarkiertem, toluidinhaltigem Polyvinylpyrrolidon ("PVP-T-131 J“) beim GoRDONTest $(1,2)$ zum Nachweis der Exsudativen Enteropatbie (3-7) stellt gewisse Stabilitätsanforderungen an dieses Präparat, um überhaupt annähernde Aussagen über einen enteralen Verlust treffen zu können. Diesen Stabilitätsanforderungen hält das Präparat nicht stand. Die Aussagekraft des Gordon-Testes mindern zwei nicht abzuschätzende Fehlermöglichkeiten; nämlich die erwähnte Instabilität des PVP-T-131 J in vivo und die problematische Korrelation zwischen enteralem Verlust der Testsubstanz und enteralem Proteinverlust (8).

Der Einball von ${ }^{131}$-Jod in das PVP: Aromatischer Einbau oder Additionsreaktion?

Die Abhängigkeit der ${ }^{131}$-Jodeinbaurate in das PVP von der Markierungsart ist erwiesen $(9,10)$. VoN GunTEN (9) und Medenwald (10) konnten durch UV-Bestrahlung einen höheren ${ }^{131}$-Jodeinbau in das PVP-T bzw. in das PVP-Molekül nachweisen, als mit der klassischen GoRDoN'schen Markierungsmethodik möglich ist. Die erste Beobachtung des höheren Aktivitätseinbaues unter UV-Bestrahlung geht dabei auf GorDON zurück. Das GoRDON'sche Markierungsverfahren basiert auf der - Úberlegung, daß unter Anwesenheit von p-Toluidin polymerisiertes PVP noch freie Aminogruppen enthalten müßte. Diese sollen sich mit Natriumnitrit in mineralsaurer Lösung diazotieren und sich ähnlich der Sandmeyer-Reaktion bei Bromiden und Chloriden gegen trägerfreies ${ }^{131}$ Jod austauschen lassen (11). GoRdoN (11) nahm daher folgende Formel für sein PVP-T-131 JProdukt an (Abb. 1).

Die Instabilität des Gordon'schen Markierungsproduktes läßt aber berechtigte Zweifel an der aromatischen Bindung des ${ }^{131} \mathrm{Jod}$ aufkommen. Offenbar kommt es
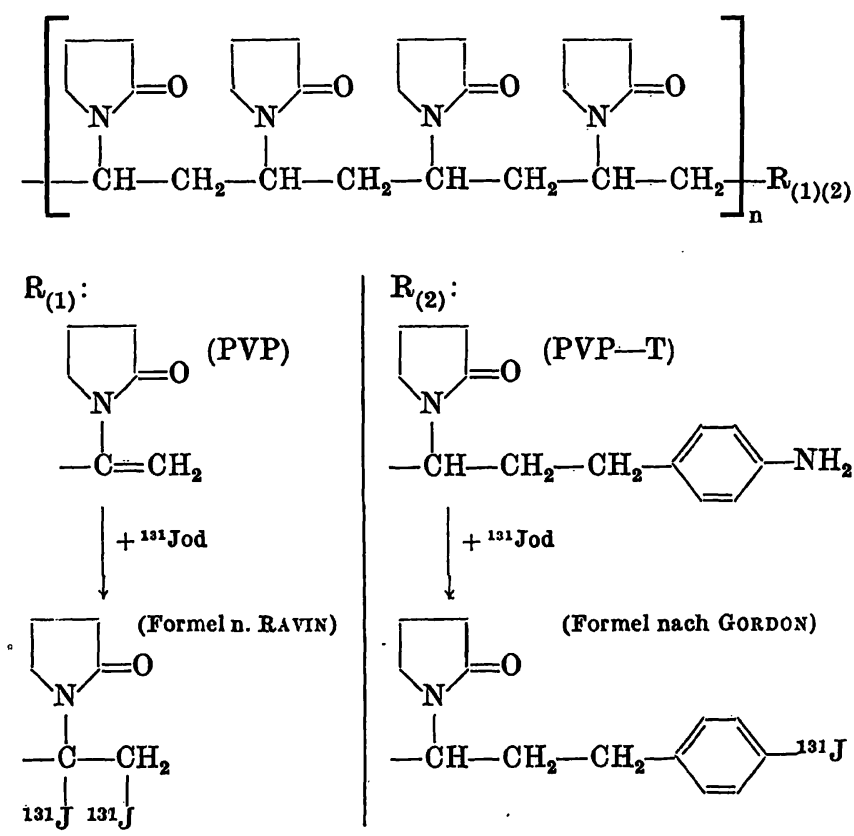

Abb. 1

Die hypothetische Formel für das PVP-T-131 J nach Gordon 
nicht zur Ausbildung von endständigen Aminogruppen, die aufgrund des Polymerisationsablaufes nach dem Radikalkettenmechanismus durch Wasserstoffperoxydkatalyse bei Kettenabbruch durch die Anwesenheit von p-Toluidin durchaus denkbar wären. Gegen eine Endgruppenmarkierung des PVP-T-Moleküls mit ${ }^{131} \mathrm{Jod}$ spricht auch die Beobachtung von Medenwald (10), der bei fraktionierter Fällung verschiedener PVP-Fraktionen an den niedermolekularen Fraktionen keine höhere Aktivität nachweisen konnte. Vermutet wird daher mit Recht, daß die Markierung des PVP-T ebenso wie die des PVP nur an Kohlenstoff-Kohlenstoff-Doppelbindungen des PVP erfolgt. Für einen gleichen Einbau des ${ }^{131}$ Jod bei beiden Präparaten sprechen auch dieselbe Thermolabilität und Alterung. Eine Einführung des ${ }^{131}$ Jod in Kohlenstoff-Kohlenstoff-Doppelbindungen nahmen schon RAviN und Mitarbeiter (zit. n. 10) an. Allerdings war die Vermutung hier naheliegender, da sie ein Präparat mit überwiegend olefinischer Kette verwendeten. Auch bei diesem Präparat dürfte die angenommene Vinylgruppe am Kettenende nur einen Teil der Aktivität tragen, da bei fraktionierter Fällung die niedermolekularen Anteile keine höhere Aktivität aufwiesen. Die Anzahl der Doppelbindungen im PVP ist abhängig vom gewählten Polymerisationsverfahren und muß sehr gering sein, denn die Markierungsverfahren zeigen eine empfindliche Beeinflussung durch geringe Mengen Trägerjod.

Kataphoreseversuche des PVP haben gezeigt (12), daß der Hauptanteil des PVP im elektrischen Feld zur Kathode wandert. Gleichzeitig wurde eine schwache anodische Wanderung beobachtet, die auf eine geringfügige Aufspaltung des Moleküls während der: Polymerisation zurückgeht. Dabei entstehen freie Carboxylbzw. enolisierte Carbonylgruppen (12). Daß es zu einem p-Toluidineinbau während der Polymerisation unter Ringaufspaltung kommt, wäre durchaus denkbar. Einen direkten Vorteil für die ${ }^{131}$.Jodmarkierung des Präparates hätte eine derartige $\mathrm{p}$-Toluidineinführung allerdings nicht (Abb. 2), aber es würde dann der gleiche Einbau-

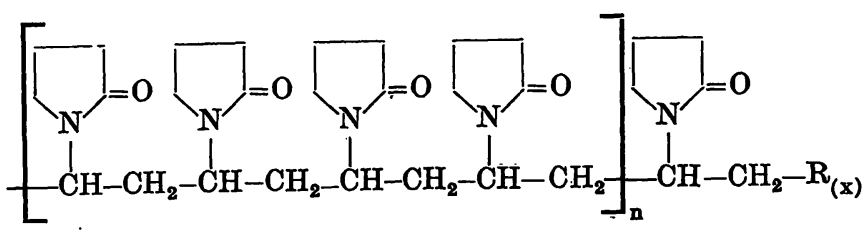<smiles>[R7]CCCCNC([R17])C</smiles>

$\mathbf{R}_{(1)}$ :

$=\mathrm{CH}_{2} \quad$ (Kettenende)

$\mathbf{R}_{(2)}:$

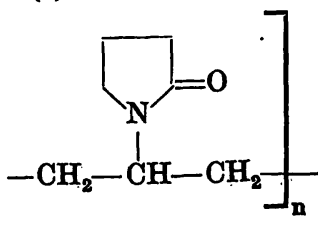

Abb. 2

Der mögliche Einbau des p-Toluidins in das PVP mechanismus des ${ }^{131} \mathrm{~J}$ wie beim PVP zugrunde liegen, wodurch die etwa gleichhohen Einbauraten zu erklären wären. - Die von uns gewählte Markierungsform erfolgte in Anlehnung an das Verfahren nach voN GUNTEN (9), wobei unter UV-Licht markiert wurde. Dabei stand uns ein Präparat der Fa. Bayer AG, Leverkusen zur Verfügung, dessen k-Wert (FIKENTSCHER-Konstante (12) 31 betrug. Das markierte Injektionspräparat wurde vor der Injektion durch Passieren einer Anionenaustauschersäule (L-150) jodfrei gemacht ( $1-2 \%$ ionogener Restjodgehalt) und durch Keimfiltration „sterilisiert".

\section{Stabilität in vitro: Die Altcrung des PVP-T-131J}

Die Instabilität des GoRDoN'schen Testpräparates in vitro wurde von uns bereits früher beschrieben (13). Der Vollständigkeit halber seien sie hier nochmals angeführt:

\begin{tabular}{|c|c|c|c|}
\hline \multirow{2}{*}{ Zeit } & \multirow{2}{*}{ Temperatur } & \multicolumn{2}{|c|}{$\%{ }^{131} \mathrm{Jod}$ gebunden } \\
\hline & & & 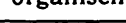 \\
\hline $20 \mathrm{Min}$. & 99 & 63,2 & 36,8 \\
\hline $30 \mathrm{Min}$. & 99 & 74,8 & 25,2 \\
\hline $45 \mathrm{Min}$. & 99 & 85,1 & 14,9 \\
\hline $0,5 d$ & 20 & 5,0 & 95,0 \\
\hline 2,0 & 20 & 11,0 & 89,0 \\
\hline 4,0 & 20 & 14,0 & 86,0 \\
\hline 9,0 & 20 & 26,0 & 74,0 \\
\hline 19,0 & 20 & 38,0 & 62,0 \\
\hline
\end{tabular}

Die Versuche wurden mit Anionenaustauschern durchgeführt und durch Dialyse bestätigt. Diese Befunde sprechen gegen eine aromatische Bindung des ${ }^{131}$ Jod und damit gegen die von GoRdon formulierte Strukturformel.

\section{Stabilität in vivo wäbrend der Körperpassage}

Die Vermutung, daß ein in vitro "genügend“ stabiles Produkt auch in vivo stabil sei (1), dürfte nicht zutreffen. Die fermentative Dejodierung war in vivo wahrscheinlich und konnte von uns durch Stuhl- und Urindialysen gesichert werden. Bei oral gegebenen PVP-T-131 J-Präparaten erfolgt diese Dejodierung offenbar in der Darmwand. Das dürfte auch die zahlreichen Mißerfolge der Stuhlinkubationen erklären. Eine Blockierung der Schilddrüse ist nicht in der Lage, eine Dejodierung des Präparates zu verhindern. Anhand der Ausscheidungsprodukte sollte die Existenz ionogenen ${ }^{131}$ Jods durch Dialyse gesichert werden.

\section{Methodik}

Die Grenzen der Trennung durch Dialyse sind bekannt. Auch bei unseren Vorversuchen konnten wir feststellen, daß etwa $10 \%$ der membrandurchlässigen Substanz innerbalb der Dialysemembran verblieb. Dieser Dialysefehler erhöht sich bei der Dialyse kleinerer Aktivitäten. Uns kam es jedoch darauf an, die Abspaltung des ${ }^{131} \mathrm{~J}$ aus dem PVP-T überhaupt nachzuweisen.

Die Dialysen wurden mit einem Dialyseschlauch der Fa. Kalle durchgeführt. Dialysiert wurde in $10 \mathrm{l}$-Bechergläsern gegen dest. Wasser, das mit einem Rührmotor in ständiger Bewegung gehalten wurde. Zur Ermittlung der maximalen Dialysedauer wurden Vorversuche mit der gleichen Aktivität durchgeführt. Dabei zeigte es sich, daß sich die Kurve des dialysierten ${ }^{131} \mathrm{Jod}$ nach 13 stdg. Dialyse einem Maximum näherte. Bei Einhaltung eines zeitlichen 


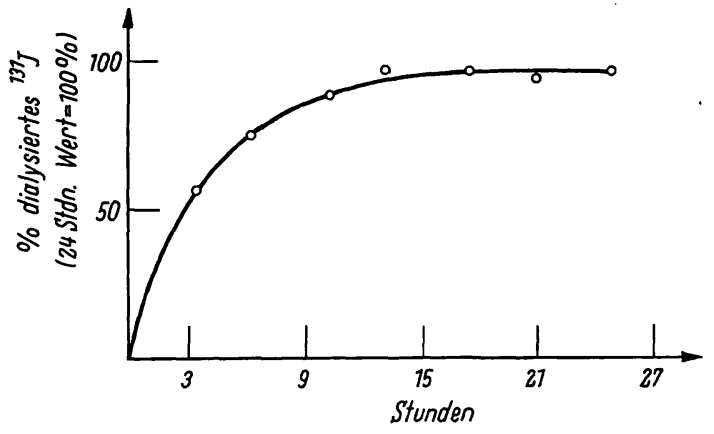

Abb. 3

Die Dialyse-Zeitkurve des ${ }^{131} \mathrm{~J}$

Sicherheitsabstandes von 7 Stdn. ergab sich eine maximale Dialysedauer von $20 \mathrm{Stdn}$. für die weiteren Dialyseversuche (Abb. 3). Die Durchführung des PVP-T_131-J-Testes erfolgte analog der bekannten Gordon'schen Testmethodik (14). Der Patient erhielt am ersten Testtag etwa $50 \mu$ C PVP-T-131 J intravenös verabfolgt. Anschließend wurden 4-5 Tage die 24-Stdn.-Fraktionen des Urins und Stuhls unter strenger Trennung beider ermittelt. Um Kontrollwerte zur Dialyse zu haben, wurde die Tagesgesamtaktivität im Urin und im Stuhl vor der Dialyse bestimmt. Während des Testverlaufes erfolgte zusätzlich eine Messung der Schilddrüsenaktivität bei blockierter Schilddrüse. Die Dialyse der Ausscheidungsprodukte erfolgte jeweils einen Tag nach der Ausscheidung. Bestimmt wurde nach 20 stdg. Dialyse:

I. die Restaktivität „R“ im Dialyseschlauch = nicht dialysabler Anteil

II. die Dialysewasseraktivität „D“ der Außen-. flüssigkeit

$=$ dialysabler

III. die Filteraktivität „,F“ der Außenflüssigkeit Anteil

Die Fraktionen I und II wurden durch Entnahme von $20 \mathrm{ml}$ Flüssigkeit in $50 \mathrm{ml}$ Bechergläsern durch Aufsetzen auf ein $2 \mathrm{~cm}$ Bohrlochkristall (Fa. Friesecke \& Höpfner, Erlangen) bestimmt. Für jede Probenserie wurde eine Vergleichslösung mit bekannter Aktivität angefertigt. Anschließend erfolgte bei der Restaktivität die Umrechnung auf die Tagesgesamtmenge, bei II (Dialysewasseraktivität) die Umrechnung auf das Gesamtvolumen an dest. Wasser unter Berücksichtigung der Abklingquote. Zur Sicherung von II erfolgte die Bestimmung III (Filteraktivität). Die Ermittlung der Filteraktivität , $\mathrm{F}^{\text {“c }}$ wurde so vorgenommen, daß etwa $20-25 \mathrm{ml}$ einer 12,5-proz. Kaliumjodidlösung nach beendeter Dialyse und Entfernung des Dialyseschlauches dem Außenwasser als Trägersubstanz zugegeben wurden. Anschließend erfolgte Fällung mit 20-proz. Silbernitratlösung. Dabei wurde sowohl radioaktives als auch nichtradioaktives Jod gefällt. Für eine gute Durchmischung mit Hilfe des Rührmotors wurde gesorgt. Anschließend Absaugen des Niederschlages mit Hilfe eines Büchnertrichters und Messung des Filters in einem $50 \mathrm{~m} l$ Becherglas durch Aufsetzen auf das Bohrlochkristall. Eine Vergleichslösung bekannter Aktivität entsprechend der Filterhöhe wurde angefertigt. Im Filtrat ließ sich keine wesentliche Aktivität mehr nachweisen. Die Beurteilung des dialysablen Anteils erfolgte grundsätzlich nach der Filteraktivität (III); diese lag gering unter der Dialysewasseraktivität (II).

Die sog. Restaktivität (I) entspricht nur zum Teil der nichtdialysablen Fraktion. Großen Anteil an ihr hat die dialysable Restfraktion (10-15\% der Außenaktivität bei Urindialysen, bei Stuhldialysen bis $50 \%$ ), die infolge des Dialysefehlers innerhalb der Membran verbleibt. Die Restaktivität (I) ist also nicht identisch mit PVP-T${ }^{131} \mathrm{~J}$, sondern setzt sich aus ${ }^{131} \mathrm{~J}$ und unzersetztem PVP-T-131 J zusammen. Die dialysable Fraktion wurde mit Hilfe zweier unterschiedlicher Arbeitsmethoden bestimmt, also Probeentnahme aus dem Außenwasser und quantitative Fällung der Außenaktivität. Sic besteht aus der ionogenen ${ }^{\mathbf{1 3 1}}$ Jod-Fraktion. Ein minimaler An- teil niedermolekularen PVP-T ${ }^{\mathbf{1 3 1}} \mathrm{J}$ im Außenwasser wäre theoretisch möglich, dürfte aber keine Rolle spielen. Auffällig war das Verhältnis Restaktivität „, $R^{c 6}$ (I) zur Filteraktivität „„F“ (III) im Urin am ersten Testtag im Vergleich $z u$ den folgenden Tagen bei intravenöser Applikation des Präparates. An diesem Tag fand sich konstant ein geringeres Verhältnis von Restaktivität zur Filteraktivität, d. h. die Restaktivität war relativ hoch. Eine unvollständige Dialyse wurde durch Kontrolldialysen ausgeschlossen. Diese erhöhte Restfraktion am ersten Testtag ließ sich nicht bei oraler Applikation des Testpräparates nachweisen. Es erscheint daher möglich, daß eine geringe Menge von unzersetztem PVP-T-131 J (etwa 10\%) ausgeschieden wird.

\section{Ergebnisse}

Urindialysen

Intravenöse Applikation (20 Urindialysen an 5 Patienten)

Bei den Urindialysen (Abb. 4) nach intravenöser Applikation des Testpräparates (PVP-T-131 J) passierten $70-80 \%$ der ausgeschiedenen Aktivität die Membran und konnten durch Probeentnahmen (D) und Fällung mit $\mathrm{AgNO}_{3}$ im Außenwasser (F) bestimmt werden. Der Dialysefehler muß bei Gesamteinschätzung des ionogenen ${ }^{131} \mathrm{~J}$-Anteils mit berücksichtigt werden, so daß der abgespaltene Aktivitätsanteil weiter ansteigt. Auf die höhere Restaktivität am ersten Ausscheidungstag war schon hingewiesen worden, so daß die Möglichkeit einer geringen Filtration von unzersetztem PVP-T-131 J durch die Nieren besteht. Die Restaktivität zeigt im Vergleich mit der Filteraktivität am 2. Tag eine fallende Tendenz, d. h. ein höheres Verhältnis. Am 3. und 4. Tag machte sich dann in zunehmendem Maße aufgrund der Kleinheit der Aktivitäten der Dialysefehler bemerkbar. Der Dialysefehler betrug am 2. Testtag, wo man mit ziemlicher Sicherheit eine völlige ${ }^{131}$-Jodabspaltung annehmen konnte, $10-15 \%$. Im allgemeinen wurde ein durchschnittlicher Dialysefehler von $15 \%$ angenommen. Diese Werte stimmen mit der Literatur überein.

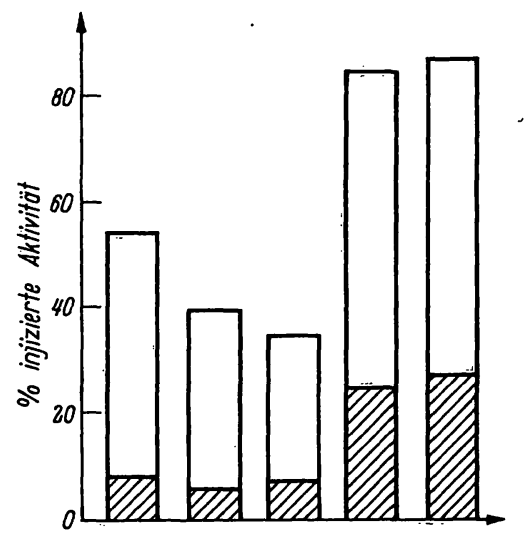

Abb. $4^{1}$ )

Das Verhältnis von dialysabler Fraktion ( ${ }^{131} \mathrm{~J}$ ) zur nichtdialysablen Fraktion (PVP-T-131 J + Rest-131 J) im Utin nach intravenöser Applikation der Testsubstanz

1) Eine Säule entspriçht vier 24-Std.-Urin- bzw. Stuhlaktivitäten von einem Patienten. Die jeweilige dialysable bzw. nichtdialysable Fraktion ist daher die Summe von vier Einzeldialysen. 


\section{Orale Applikation ( 8 Urindialysen an 2 Patienten)}

Die orale Applikation des PVP-T-131 J dürfte insofern interessant sein, als sie gewisse Maßstäbe für die Stabilität der makromolckularen Markierungsverbindung zuläßt. Nach Schefrener (15) und anderen Autoren kommt es zu keiner Resorption von PVP aus dem Magen-DarmKanal. Andererseits wäre cine geringe Resorption ebenso wie die bekannte physiologische Exkretion durchaus denkbar, zumal die Versuche von Volkheiner (16) die Möglichkeit der Resorption von Makromolekülen gezcigt haben. Unsere Urindialysen nach oraler Applikation (Abb. 5) der Testsubstanz zeigten jedoch, daß es sich bei der im Urin ausgeschiedenen Aktivität um ionogenes ${ }^{131}$ Jod handelt. Dabei wäre zu diskutieren, ob primär eine geringe Menge PVP-T-131 J zur Resorption kommt und im Körper „,dejodiert" wird, oder ob nur ionogenes ${ }^{131} \mathrm{Jod}$ resorbiert wird. Als möglicher Abspaltungsort bei oraler Gabe wird die Magen-Darmschleimhaut angenommen, was auch die vielen ergebnislosen Fäkalinkubationen erklären dürfte. Auffällig war bei der oralen Applikationsart das andere Verhältnis von im Beutel verbliebener Restaktivität und dialysierter Aktivität (F) im Urin.

\section{Beispiele}

Urindialyse nach oraler Verabfolgung von 27,6 $\mu$ C PVP-T-1''J

\begin{tabular}{lcc}
\hline & $\begin{array}{c}\text { Restaktivität im Beutel } \\
\text { nach 20stdg. } \\
\text { Dialyse }\end{array}$ & $\begin{array}{c}\text { Außenaktivităt nach } \\
\text { 20stdg. Dialyse } \\
\text { (Filteraktivität) }\end{array}$ \\
\hline 1. Tag: & $0,61 \mu \mathrm{C}(2,2 \%)$ & $7,00 \mu \mathrm{C}(24,6 \%)$ \\
2. Tag: & $0,35 \mu \mathrm{C}(1,25 \%)$ & $5,89 \mu \mathrm{C}(21,3 \%)$ \\
3. Tag: & $0,14 \mu \mathrm{C}(0,51 \%)$ & $2,76 \mu \mathrm{C}(10,0 \%)$ \\
4. Tag: & $0,16 \mu \mathrm{C}(0,58 \%)$ & $1,07 \mu \mathrm{C}(3,9 \%)$ \\
\hline
\end{tabular}

Urindialyse nach intravenöser Injektion von 49,2 $\mu \mathrm{C}$ PVP-T-131 J

\begin{tabular}{ccc}
\hline & $\begin{array}{c}\text { Restaktivität im Beutel } \\
\text { nach 20-stdg. } \\
\text { Dialyse }\end{array}$ & $\begin{array}{c}\text { Außenaktivität nach } \\
\text { 20-stdg. Dialyse } \\
\text { (Filteraktivität) }\end{array}$ \\
\hline 1. Tag: & $2,21 \mu \mathrm{C}(4,5 \%)$ & $5,71 \mu \mathrm{C}(11,61 \%)$ \\
2. Tag: & $0,56 \mu \mathrm{C}(1,15 \%)$ & $4,00 \mu \mathrm{C}(8,06 \%)$ \\
3. Tag: & $0,26 \mu \mathrm{C}(0,54 \%)$ & $2,32 \mu \mathrm{C}(4,72 \%)$ \\
4. Tag: & $0,20 \mu \mathrm{C}(0,4 \%)$ & $1,4 \mu \mathrm{C}(2,8 \%)$ \\
\hline
\end{tabular}

Die bei der intravenösen Applikation nachweisbare höhere Restaktivität am 1. Ausscheidungstag läßt sich bei oraler Applikation nicht nachweisen. Die Ausscheidungsphase zeigt einen flacheren und bei Umrechnung auf äquivalente Urinmengen einen fast linearen Ausscheidungsverlauf. Auch die Serumkurve verlief flacher und zeigte durchschnittlich 2 Stdn. nach oraler Applikation eine $50 \%$ niedrigere Serumkonzentration.

\section{Stuhldialysen}

\section{Intravenöse Applikation (20 Stubldialysen an 5 Patienten)}

Nach BeNNHold (17) bestimmt man im Stuhl reine PVP-T-131 J-Aktivität; auch OEFF ist der Meinung, daß evtl. zur Ausscheidung kommendes ${ }^{131} \mathrm{~J}$ sehr schnell rückresorbiert wird. Diese Angaben konnten wir nicht bestätigen. Die dialysable ionogene Fraktion (Abb. 6) schwankte zwar bei den einzelnen Patienten erheblich, ließ sich aber einwandfrei sichern.
Beispiel

Stuhldialyse nach intravenöser Applikation von 45,1 $\mu \mathrm{C}$ PVP-T-1.1 J

\begin{tabular}{|c|c|c|}
\hline & $\begin{array}{c}\text { Restaktivität im Beutel } \\
\text { nach } 20 \text { stdg. } \\
\text { Dialyse }\end{array}$ & $\begin{array}{l}\text { Außenaktivität nach } \\
20 \text { stdg. Dialyse } \\
\text { (Filteraktivität) }\end{array}$ \\
\hline $\begin{array}{l}\text { 1. Tag: } \\
\text { 2. Tag: } \\
\text { 3. Tag: } \\
\text { 4. Tag: }\end{array}$ & 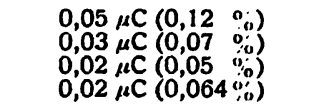 & $\begin{array}{l}0,01 \mu \mathrm{C}(0,02 \%) \\
0,005 \mu \mathrm{C}(0,01 \%) \\
0,03 \mu \mathrm{c}(0,06 \%) \\
0,006 \mu \mathrm{c}(0,014, \%)\end{array}$ \\
\hline
\end{tabular}

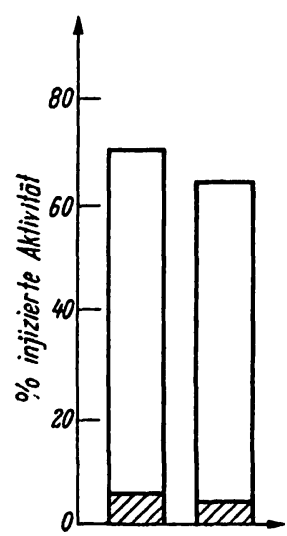

Abb. $5^{1}$

Oralc Applikation des Präparates und Trennung von ${ }^{131} \mathrm{~J}$ und PVP-T_-131 J durch Dialyse des Urins

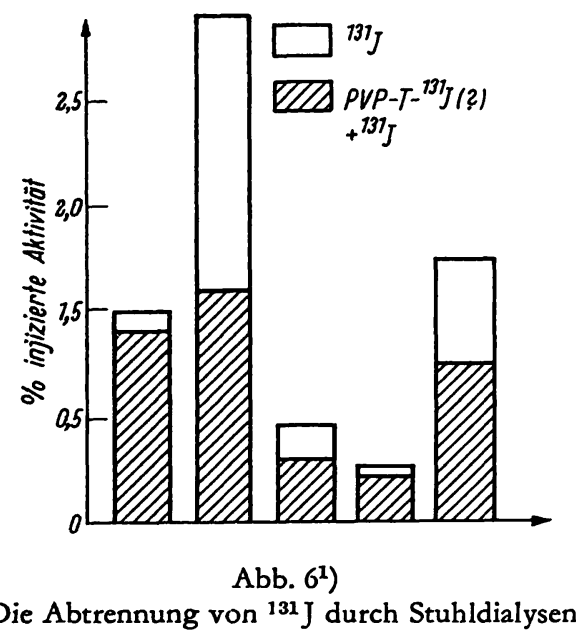

Sie bewegte sich in den Grenzen von 8-56\% der Gesamtstuhlaktivität (Pat. mit 1-4\% Stuhlausscheidung). Die gefundenen Werte dürften Mindestwerten entsprechen, da die ionogene Jodrückresorption u. a. vom Funktionszustand der Schilddrüse abhängig ist und individuell variieren kann. Dazu kommt ein unterschiedlicher Dialyseerfolg bei kleinen Aktivitäten. Ob unzersetztes PVP-T-131 J überhaupt ausgeschieden wird, konnte nicht endgültig geklärt werden. Somit kann man aufgrund dieser Ergebnisse keine Parallelität zwischen Aktivitātsübertritt in den Darm und enteralem Eiweißverlust erwarten.

Verwendung von Anionenaustauschern

Von JEEJEEBHOY (18) erfolgte erstmalig die Verwendung von Anionenaustauschern zur Bindung des abgespaltenen ionogenen ${ }^{131} \mathrm{~J}$ im Darm beim ${ }^{131} \mathrm{~J}$-Albumintest. In Analogie dazu suchten wir das vom PVP-T-131 J abgespaltene ${ }^{131} \mathrm{~J}$ in vivo an Amberlite zu binden. Beden- 
ken gegen die Amberlite-Technik beim Albumin- ${ }^{131} \mathrm{~J}-$ Test sind erst in jüngster Zeit wieder geäußert worden, da diese Technik nur semiquantitative Ergebnisse liefert. ChAPMAN und Mitarbeiter (19) konnten durch Versuche sichern, da $\beta$ an Amberlite gebundenes ${ }^{131} \mathrm{~J}$ nach Verabreichung per os zu einem nicht unwesentlichen Teil im Urin erscheint, umgekehrt aber ein großer Teil von intravenös appliziertem $\mathrm{Na}^{131} \mathrm{~J}$ im Stuhl auftritt und dort an Amberlite gebunden wird. Sie beobachteten eine enterale Ausscheidung von $10-47 \%$ der injizierten Aktivität und bestätigten mit ihren Versuchen auch die von uns ausgesprochene Vermutung (8), daß es bei gastrointestinalen I.äsionen zu einer höheren ${ }^{131}$ Jodausscheidung korm.m. Unsere Versuche mit der AmberliteTechnik bei PVP-T-131 J Applikation zeigten eine verminderte Urinausscheidung und eine höhere Gesamtaktivität im Stuhl als bei anderen gesunden Personen ohne Amberlite-Gabe.

Zusammenfassend muß festgestellt werden: Die PVP-T${ }^{131} \mathrm{~J}$ - bzw. die PVP_131 J-Präparate sind in vitro, besonders jedoch in vivo instabil. Die leichte Abspaltbarkeit erklärt sich aus der Addition von ${ }^{131}$ Jod an Doppel- bindungen im PVP-Molekül. Die von GoRDon formulierte aromatische Jodbindung liegt mit großer Wahrscheinlichkeit nicht vor. Eine Parallelität zwischen enteralem Verlust von Eiweiß und enteralem PVP-T-131 JVerlust ist-somit nicht zu erwarten. Die im Urin erscheinende Aktivität entspricht zu 90\% reinem ${ }^{131} \mathrm{~J}$. Am ersten Testtag wäre aufgrund der hohen Restaktivität im Beutel nach beendeter Dialyse eine geringe PVP-T-131 J-Ausscheidung möglich (etwa 10\%). Der Träger der fäkalen Aktivität ist zu mindestens 30 bis $50 \%$ ebenfalls das ionogene abgespaltene ${ }^{131} \mathrm{Jod}$. Aufgrund der Methodik konnte kein höherer Anteil abgetrennt werden. Wahrscheinlich - in Verhindung mit Urindialysen - tritt kaum PVP-T-131 J über. - CHAPMAN (19) wies eiren erhöhten ${ }^{131}$ Jodübertritt bei gastrointestinalen Läsionen nach. In diesem Zusammenhang wäre die Frage aufzuwerfen, ob ein pathologischer Gordon-Test (enterale Aktivität über $1,1 \%$ der injizierten Aktivität) nicht durch einen höheren Verlust (und verminderte Rückresorption) von aus dem PVP-T${ }^{131} \mathrm{~J}$ abgespaltenen ${ }^{131}$ Jod zustande kommt und so eine PVP-T-131 J Ausscheidung vortäuscht.

\section{Literatur}

1. Aebersold, J., Helv. med. Acta 32, 227 (1965). - 2. Jarnum, S., Scand. J. clin. Lab. Invest. 13, 447 (1961). - 3. Barandun, S., J. Aebersold, R. Bianchi, R. Kluthe, G. von Muralt, G. Poretri und G. Riva, Schweiz. med. Wschr. 90, 1458 (1960). 4. Martini, G. A., W. Dölle, F. Petersen, U. Treske und G. STrohmeyer, Internist (Berl.) 4, 197 (1963). - 5. SChwartz, M. und S. Jarnum, Lancet I, 327 (1959). - 6. Seige, K., H. HarTManN, H. КосH, J. Löbe und H.-J. Lieschke, Dtsch. Zschr. Verdauungskrkh. 25, 237 (1965). - 7. SeIGE, K., H. HARTMANN, H.-J. LieschKe, J. LÖBE und H. КосH, Zschr. ges. inn. Med. 20, 669 (1965). - 8. Seige, K., H. Koch, J. Löbe und H. HarTMANN, Zschr. ges.inn.Med. 19, H. 24 (Tagungsberichte), 223 (1964). - 9. von Gunten, H. R., H. Hügli und P. Tempus, Experientia
(Basel) 17, 299 (1961). - 10. Medenwald, H., Z. Naturforsch. 17b, 113 (1962). - 11. Gordon, R. S., J. Polymer. Sci. 31, 191 (1.958). - 12. REPpE, W., Polyvinyl pyrrolidon," Verlag Chemie, Weinheim (1954). - 13. KoCH, H., K. SEIGE, J. LÖHE und H. Hartmann, Kernenergie 7, 507-509 (1964). - 14. Gordon, R. S., Lançet $I$, 325 (1959). - 15. SchefFner, D., Dissert. Heidelberg 1955. - 16. Volkheimer, G. und F.-H. Schulz, Zschr. ges. inn. Med. 20, 653 (1965). - 17.Bennhold, H. und H. Ott, Med. Klin. 57, 814 (1962). - 18. JeEjeEbhoy, K.H. und N. F. CoghrLL, Gut 2, 123 (1961). - 19. Chapman, A., G. H. Jefrries und M. H. SLEISEnger, „Physiology and pathoihysiology of plasma protein metabolism" S. 27, H. Huber-Verlag, Bern-Stuttgart (1965).

Dr. med. H.-J. Lieschke

X 701 Leipzig, Philipp-Rosenthal-Straße 